ORIGINAL ARTICLE

\title{
Relation between foot arch index and ankle strength in elite gymnasts: a preliminary study
}

\author{
S T Aydog, L Özçakar, O Tetik, H A Demirel, Z Hasçelik, M N Doral
}

Br J Sports Med 2005;39:e13 (http://www.bjsportmed.com/cgi/content/full/39/3/el3). doi: 10.1136/bjsm.2004.011627

See end of article for authors' affiliations

.......................

Correspondence to: Dr Aydog, Department of Sports Medicine,

Hacettepe University

Medical School, Ankara

06100, Turkey; taydog@

hacettepe.edu.tr

Accepted 6 April 2004
Background: Gymnasts usually start intensive training from early childhood. The impact of such strenuous training on the musculoskeletal system is not clear.

Objectives: To evaluate the relation between muscle strength of the ankle joint and foot structure in gymnasts.

Methods: The study population comprised 20 high level male gymnasts and 17 non-athletic healthy male controls. Arch indices were measured using a podoscope. Ankle plantar/dorsiflexion and eversion/ inversion strengths were measured using a Biodex 3 dynamometer within the protocol of concentric/ concentric five repetitions at $30 \%$ velocity.

Results: The mean arch index of the right and left foot of the gymnasts and the controls were respectively: 31.4 (29.1), 34.01 (34.65); 60.01 (30.3), 63.75 (32.27). Both the arch indices and the ankle dorsiflexion strengths were lower in the gymnasts. Although no correlation was found between strength and arch index in the control group, a significant correlation was observed between eversion strengths and arch indices of the gymnasts $(r=0.41, p=0.02)$.

Conclusions: Whether or not the findings indicate sport specific adaptation or less training of the ankle dorsiflexors, prospective data are required to elucidate the tendency for pes cavus in gymnasts, for whom stabilisation of the foot is a priority.
A s sport becomes more popular, the age at which individuals start tends to decrease. Subjects who have undergone intense training since early childhood may show adaptive changes in body structures when they reach adulthood. Although there is a lack of strong evidence, a few studies have shown sport specific changes in the musculoskeletal system. ${ }^{1-3}$ The best example is the increased external rotation and decreased internal rotation in the shoulders of tennis players. ${ }^{1}$ Other examples are long distance runners and skiers, in whom a tendency towards pes planus has been observed..$^{2}$ Bearing in mind the heavy training programmes of gymnasts starting from early childhood, it seems reasonable that these athletes will also display musculoskeletal changes.

The foot arch is a structure that changes with age. ${ }^{4}$ Although the main parameters that cause this changing pattern have not been clearly identified, the muscles in the foot are known to affect the progression of the foot arch. The relevant literature does not contain sufficient prospective studies, but pes planus reported in elderly patients with rupture of the tibialis posterior tendon shows the effect of muscular problems on the foot arch. ${ }^{5}$ The altered rear foot pronation after eight weeks of isokinetic ankle inversion and eversion exercises, ${ }^{6}$ and the development of pes planus in $10-$ 11 year old athletes who perform 18-30 hours of training a week $^{3}$ are also other noteworthy studies. Thus it seems likely that exercise of the muscles connected with the ankle joint will affect the foot arch.

In a previous study ${ }^{7}$ comparing athletes from five different sports (handball, weightlifting, football, wrestling, gymnastics) and controls, we found that the gymnasts had lower arch indices. Although we cannot say that these differences were sport specific, we have shown once again that they are different. In this study, our aim was to evaluate the muscular strength of the ankle joints of gymnasts and to seek a relation between the arch indices and this muscle strength.

\section{MATERIALS AND METHODS}

The study population comprised 22 male elite gymnasts and 17 non-athletic male volunteers as controls. Age, height, weight, and age at onset of training were recorded. Two gymnasts who had been treated with a cast for foot and ankle fractures or dislocations were excluded from the study.

\section{Measurements of sole arch index (AI)}

Subjects were asked to stand still on the podoscope. Digital still camera images of both soles in the podoscope were transferred to a computer. From the stored images, AI was calculated by dividing the narrowest part of the sole by the widest part of the heel, then multiplying the ratio by $100 .{ }^{4}$ All calculations were performed by the same clinician. The clinician calculated another 30 foot AIs twice, with a one week interval between measurements, to determine intraobserver reliability (intraclass correlation coefficient was 0.975).

\section{Isokinetic muscle testing}

Muscle strength of the ankle joints was measured using a Biodex 3 dynamometer (Biodex Medical System, Inc, New York, New York, USA). All subjects were tested without shoes. Each subject was seated in the adjustable chair of the dynamometer, and the leg being tested was elevated by a

Table 1 Anthropometric characteristics of the gymnasts and control group

\begin{tabular}{llll}
\hline & Gymnasts $(\mathbf{n}=20)$ & Controls $(\mathbf{n}=17)$ & $\mathbf{p}$ Value \\
\hline Age (years) & $22.9(3.78)$ & $22.80(3.03)$ & 0.80 \\
Weight $(\mathrm{kg})$ & $63.24(5.61)$ & $71.65(13.06)$ & 0.07 \\
Height $(\mathrm{cm})$ & $170.29(5.63)$ & $176.56(8.99)$ & 0.02 \\
Right Al & $31.4(29.04)$ & $60.01(30.30)$ & 0.01 \\
Left Al & $34.01(34.65)$ & $63.75(32.27)$ & 0.04 \\
\hline
\end{tabular}

Values are mean (SD).

$\mathrm{Al}$, Arch index. 
support arm under the knee. Two diagonal standard straps stabilised the trunk and one strap secured the hip. The arms were crossed over the chest, and the contralateral foot was placed on a support arm attached to the chair. The subject's ankle was placed on the foot plate, and the foot was secured with two straps. For each subject, the attachments of the dynamometer were readjusted so that centre of motion of the lever arm was aligned as accurately as possible with the slightly changing axis of the joint. The stability of the foot on the foot plate was enhanced with a rubber heel cup, and adjustable thermoplastic stays were positioned on the medial and lateral borders of the foot. The dynamometer orientation, tilt, and seat orientations were kept at $90^{\circ}, 0^{\circ}$, and $90^{\circ}$ during plantar/dorsiflexion, and at $0^{\circ}, 50^{\circ}$, and $90^{\circ}$ during eversion/ inversion. The end range setting was standardised for all subjects from $40^{\circ}$ eversion to $45^{\circ}$ inversion and from $30^{\circ}$ dorsiflexion to $40^{\circ}$ plantarflexion. Each subject performed three to five submaximal warm up repetitions before each test. Then bilateral isokinetic (concentric/concentric) ankle plantar/dorsiflexion and eversion/inversion measurements were performed within the protocol of $30 \%$ s (five repetitions). Between the two sessions, the subjects had a one minute period of rest. Vocal encouragement during the tests was consistent and standardised. Peak torque/body weight and agonist/antagonist ratios were obtained.

Social package for statistical sciences 10.0 was used for statistical analysis. As the sole AIs and values for ankle muscle group strength did not show a normal distribution, we applied non-parametric Mann-Whitney $U$ tests for comparison of the means. Spearman's rank correlation test was used for correlation analysis. The significance level was accepted as $\mathrm{p}<0.05$.

\section{RESULTS}

The ages of the gymnasts ranged between 18 and 30 years (mean (SDS) $22.9(3.78)$ ) and that of the non-athletic controls between 18 and 28 years $(22.8(3.03))$. The age at which sports training was started was 7.3 (1.44) years. Table 1 summarises the basic data on the gymnasts and controls. No significant difference was found in age and weight between the groups but the gymnasts were significantly shorter than the controls $(p=0.02)$. Both sole AIs of the gymnasts were significantly lower than the AIs of the controls $(p<0.05)$. Sole AIs were found to correlate significantly between the two feet in both groups (gymnasts $r=0.95, \mathrm{p}=0.00$; controls $r=0.98, \mathrm{p}=0.00)$.

Table 2 summarises the isokinetic test results for both groups. The dorsiflexion muscle strength was significantly lower in both feet of the gymnasts than in the control group $(\mathrm{p}=0.00, \mathrm{p}=0.00)$. Table 3 gives the correlation analysis between the AIs and muscle strength values. No correlation

\begin{tabular}{llll} 
Table 2 & Isokinetic test results & (peak torque/ body weight) \\
\hline & Gymnasts & Control & p Value \\
\hline Right DF & $36.79(11.66)$ & $47.71(9.05)$ & 0.00 \\
Right PF & $182.45(55.53)$ & $191.01(43.58)$ & 0.68 \\
Right DF/PF & $23.34(15.85)$ & $26.24(7.72)$ & 0.03 \\
Left DF & $46.53(42.14)$ & $51.81(22.99)$ & 0.00 \\
Left PF & $189.99(44.36)$ & $185.84(35.24)$ & 0.54 \\
Left DF/PF & $25.03(19.10)$ & $29.20(17.23)$ & 0.03 \\
Right Ever & $52.04(12.64)$ & $59.52(16.12)$ & 0.23 \\
Right Inv & $46.10(11.82)$ & $48.05(14.76)$ & 0.68 \\
Right Ever/Inv & $117.51(35.76)$ & $128.49(31.70)$ & 0.23 \\
Left Ever & $51.44(14.39)$ & $59.87(18.44)$ & 0.31 \\
Left Inv & $47.52(10.70)$ & $45.43(9.06)$ & 0.71 \\
Left Ever/Inv & $115.57(35.36)$ & $134.92(46.35)$ & 0.20 \\
\hline
\end{tabular}

Values are mean (SD).

DF, Dorsiflexion; PF, plantarflexion; Ever, eversion; Inv, inversion.
Table 3 Correlation analysis between arch indices (Als) and ankle strength measurements

\begin{tabular}{|c|c|c|c|c|c|c|}
\hline & PF & DF & $\mathrm{PF} / \mathrm{DF}$ & Inv & Ever & Ever/lnv \\
\hline \multicolumn{7}{|l|}{$\mathrm{Al}$ in gymnasts } \\
\hline$r$ & -0.24 & -0.11 & 0.24 & 0.30 & 0.41 & 0.14 \\
\hline $\mathrm{p}$ & 0.16 & 0.54 & 0.18 & 0.08 & 0.02 & 0.42 \\
\hline \multicolumn{7}{|l|}{$\mathrm{Al}$ in controls } \\
\hline$r$ & -0.09 & -0.06 & 0.08 & 0.08 & 0.04 & -0.03 \\
\hline $\mathrm{p}$ & 0.62 & 0.74 & 0.67 & 0.65 & 0.81 & 0.85 \\
\hline
\end{tabular}

was found in the control group, but AI correlated with eversion strength in the gymnasts $(r=0.41, \mathrm{p}=0.02)$.

\section{DISCUSSION}

High level athletes are usually exposed to intense training since early childhood. Although certain anthropometric features are advantageous in some sports, repetition of certain exercises over a long time may also cause sport specific adaptations in the body. To hit the ball harder, training for tennis players includes increased external rotation of the shoulder, resulting in increased pre-tensioning of the internal rotator muscles of the shoulder. These acquired changes (increased shoulder external rotation and decreased internal rotation) are not only adaptations but also provide advantages to the player. ${ }^{1}$ The musculoskeletal alterations involved have not been well described, but it seems likely that similar adaptations occur in other sports that entail intense training before complete ossification of the skeleton takes place. In this study, the AIs and ankle dorsiflexor strengths of the gymnasts were found to be bilaterally lower than those of the normal healthy controls. Moreover, the AIs of the gymnasts were found to correlate significantly with the eversion strengths of their ankles.

The arch of the foot changes significantly throughout life. Staheli et $a l^{4}$ found that the medial longitudinal arch has an undulating pattern according to age and AI. The AI is about 1 (range 0.7-1.35) at 1 year of age, reducing to a minimum of 0.6 (range 0.3-0.9) at 12-14 years of age, before increasing to 0.8 (range $0.3-1.1$ ) at older ages. ${ }^{48}$ Although the underlying causes have yet to be illuminated, it has been suggested that the AI might show variations in people who participate in sports such as running, alpine skiing, football, and tennis. ${ }^{23}$ In a previous study, ${ }^{7}$ we found that gymnasts have lower AIs than handball players, football players, weightlifters, wrestlers, and non-athletic men. Both that study and the current one were cross sectional, thus we cannot conclude that this difference is gymnastic specific; however, the AIs of the gymnasts have been confirmed to be different.

To the best of our knowledge, there are no studies of the effect of exercise on the AI in the literature. However, the effect of exercise on the rear foot has been reported. ${ }^{6}$ In that study Feltner et al observed that the pronation of the rear foot changed in people who performed an eight week course of isokinetic eversion/inversion exercises. This may shed light on the possible contribution of exercise to the unstable structure of the AI.

There is only one study on gymnasts in which muscle strength of the ankle joint has been assessed. ${ }^{9}$ Plantar/ dorsiflexion measurements were obtained for 23 athletes, seven of whom were gymnasts. The muscle strengths of the gymnasts were lower than those of the cyclists, but no different from the controls. Owing to the small number of subjects, the results of the study may not be fully conclusive. We evaluated both plantar/dorsiflexion and eversion/inversion strengths in comparison with non-athletic healthy subjects, and only the dorsiflexors were found to be weaker. 
What is already known on this topic

It is known that intensive sport training started at an early age can result in musculoskeletal changes, such as the increased external rotation and decreased internal rotation seen in the shoulders of tennis players.

\section{What this study adds}

The structure and musculature of the foot and ankle is different in gymnasts from non-athletic healthy controls. No firm conclusions on the reasons for this could be drawn.

We believe that this difference cannot be due to chance alone and may stem from either insufficient dorsiflexor strengthening or sport specific adaptation. Although our study included more gymnasts than the above study, it is still not enough to draw conclusions on the cause. However, we should mention that all of our male gymnasts, older than 18 years of age, who have attended national team selections, were included in the study. Prospective studies rather than cross sectional ones are needed to clarify the sport specific adaptation of the ankle dorsiflexors of gymnasts. If inadequate dorsiflexor strengthening is responsible, this is important, as this muscle group is crucial for ankle stabilisation, which is a prerequisite in gymnastics. It would then be wise to recommend more dorsiflexion exercises for these athletes; however, if it is an adaptation, performance might be adversely affected. Thus prospective data are definitely required to establish a rational approach to ankle exercises for gymnasts.

Certain muscles in the foot and ankle either depress or support the sole arch, and their insufficiency may result in changes in the sole. For example, posterior tibial tendon ruptures and tenosynovitis result in flat foot. ${ }^{5}$ The posterior tibial, peroneus brevis/longus, flexor hallucis longus, flexor digitorum longus, and abductor hallucis longus muscles support the formation of the medial longitudinal arch, whereas the extensor hallucis longus and tibialis anterior muscles have a depressing effect on this arch. ${ }^{10}{ }^{11}$ Exercises for flat foot and pes cavus, with the exception of bony problems (rigid pes planus) such as talocalcaneal fusion, include stretching and strengthening of the intrinsic and extrinsic muscle groups. Generally, these exercises are accepted as symptomatic therapeutic modalities. ${ }^{12}{ }^{13}$ In our study, the weaker dorsiflexion strength is related to the tibialis anterior, extensor digitorum longus, and extensor hallucis longus muscles. These muscles are known to depress the foot arch, ${ }^{10}{ }^{11}$ increasing the AI, and the results of our study agree with this. The evertors of the ankle are known to support the foot $\operatorname{arch}^{10}$ however, in contrast with this, we found a correlation between the AIs and eversion strengths in our study. Although the evertors in the control group did not differ from the those of the gymnasts, the lack of a similar correlation in the non-athletic men may support the existence of a relation between AI and ankle muscle strength in athletes. Together with the effects of exercise on rear foot pronation, ${ }^{6}$ our data-that is, the correlation between eversion strength and AI-may suggest that exercise affects the structure of the foot. Once again, we admit that the number of gymnasts in our study may not support such a robust deduction.

In conclusion, we have found that bilateral foot AIs and ankle dorsiflexion muscle strengths are lower in gymnasts than in non-athletic healthy controls, and there is a significant correlation between the AIs of gymnasts and their eversion strengths. These findings may reveal a sport specific adaptation; however, prospective data on the effects of strengthening the dorsiflexors on the AI and gymnastic performance are required.

\section{Authors' affiliations}

S T Aydog, L Özçakar, O Tetik, H A Demirel, Z Hasçelik, M N Doral, Departments of Sports Medicine, Physical Medicine and Rehabilitation and Orthopedics, Hacettepe University Medical School, Ankara, Turkey

Competing interests: none declared

\section{REFERENCES}

1 Hawkins RJ, Mohtadi N. Rotator cuff problems in athletes. In: Delee JC, Drez D, eds. Orthopaedic sports medicine. Philadelphia: WB Saunders, 1994:631-2.

2 Klingele J, Hoppeler $\mathrm{H}$, Biedert R. Statistical deviations in high-performance athletes. Schweiz Z Sportmed 1993;41:55-62.

3 Volkov BM. Influence of considerable athletic training on the foot condition of young athletes at a boarding school with a cross-section of sports [abstract] Arkh Anat Gistol Embriol 1977;72:32-4.

4 Staheli TS, Chew DA, Corbett $M$. The longitudinal arch. A survey of eight hundred and eighty-two feet in normal children and adults. J Bone Joint Surg [Am] 1987;69:426-8.

5 Solomon L, Warwick D, Nayagam S. The ankle and foot. In: Apley's system of orthopaedics and fractures. London: Arnold Publishers, 2001:495.

6 Feltner ME, MacRae HS, MacRae PG, et al. Strength training effects on rearfoot motion in running. Med Sci Sports Exerc 1994;26:1021-7.

7 Aydog ST, Tetik O, Demirel AH, et al. Differences in sole arch indices in various sports. Br J Sports Med 2005;39:e5.

8 Forriol F, Pascual J. Footprint analysis between three and seventeen years of age. Foot Ankle 1990;11:101-4.

9 So CH, Siu TO, Chan KM, et al. Isokinetic profile of dorsiflexors and plantar flexors of the ankle: a comparative study of elite versus untrained subjects. Br J Sports Med 1994;28:25-30.

10 Kapandji IA. Lower limb. In: The physiology of the joints. Edinburgh: Churchill Livingstone, 1976:202-5.

11 American Academy of Orthopaedic Surgeons. The foot. In: Athletic training and sports medicine. Rosemont, IL: American Academy of Orthopaedic Surgeons, 1991:425-32.

12 Hsu JD, Mann DC, Imbus CE. Pes cavus. In: Jahss MH, eds. Disorders of the foot and ankle. Philadelphia: WB Saunders, 1992:881-2.

13 Rose GK. Pes planus. In: Jahss MH, eds. Disorders of the foot and ankle. Philadelphia: WB Saunders, 1992:907-19. 